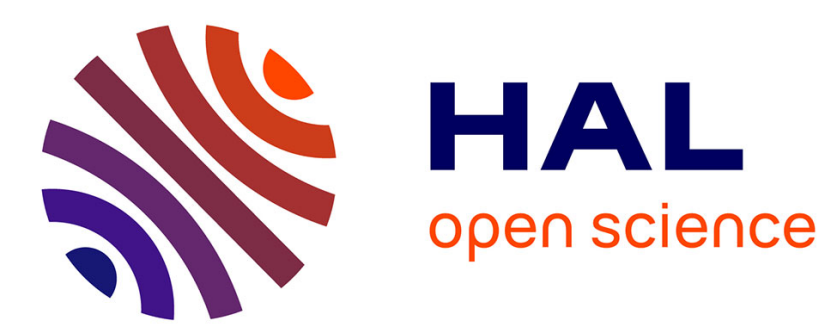

\title{
Thermomechanical Couplings and Scale Transitions in Mechanics of Materials
}

\author{
R. Peyroux, Catherine Mabru
}

\section{To cite this version:}

R. Peyroux, Catherine Mabru. Thermomechanical Couplings and Scale Transitions in Mechanics of Materials. Michel Frémond; Franco Maceri. Novel Approaches in Civil Engineering, Springer, pp.233251, 2004, 10.1007/978-3-540-45287-4_19. hal-03342803

\section{HAL Id: hal-03342803 https://hal.science/hal-03342803}

Submitted on 14 Sep 2021

HAL is a multi-disciplinary open access archive for the deposit and dissemination of scientific research documents, whether they are published or not. The documents may come from teaching and research institutions in France or abroad, or from public or private research centers.
L'archive ouverte pluridisciplinaire HAL, est destinée au dépôt et à la diffusion de documents scientifiques de niveau recherche, publiés ou non, émanant des établissements d'enseignement et de recherche français ou étrangers, des laboratoires publics ou privés. 


\title{
Thermomechanical Couplings and Scale Transitions in Mechanics of Materials
}

\author{
Robert Peyroux, Catherine Mabru
}

Laboratoire de Mécanique et Génie Civil, cc 048, Université Montpellier II, F-34095 Montpellier Cedex 5, France

\section{Introduction}

Mechanics of Materials has experienced this last decade a considerable expansion. The widening, to non strictly mechanic sciences, of available knowledge and data about the material behaviour has permitted to get a global view of the phenomena accompanying the deformation processes. The use of innovative experimental techniques, the writing of consistent theoretical framework and the recourse to high-performance numerical methods allow to analyse, to understand and to simulate the materials behaviour. Two research areas are particularly active these last years. On the one hand, the analysis of the microstructure of materials reveals the phenomena associated with the deformation process, and the use of scale transition techniques permits to integrate this description and to derive a more valuable macroscopic modelling. On the other hand, these phenomena often require variables that complete the classical displacements, strain or efforts of the mechanics. The taking into account of a temperature or a volumic fraction of phase in the constitutive equations implies the modelling of couplings between variables and the derivation of an adapted framework. The interest of this approach is again to get a more valuable description of the material behaviour, and also to use these additional variables as real tracers of the deformation process of the material.

In this paper, we first present the classical framework of Thermodynamics of Irreversible Processes, and more particularly the so-called Generalised Standard Materials approach, putting the emphasis on the modelling of thermomechanical couplings and their experimental estimations. Then, after a brief review of the various works carried out in the team Thermomechanics of Materials of the LMGC, we will detail two applications, one dealing with a multiscale analysis of the thermomechanical behaviour of Shape Memory Alloys, and the other connected with thermal and dissipative effects associated with fatigue of metallic alloys. 


\section{A convenient thermomechanical framework}

A convenient thermodynamic framework is the Continuum Thermodynamics (Germain, 1973, Germain, 1983), that postulates the local state axiom and considers the material both as a continuum and as a thermodynamic system described by a set of state variables. More precisely, we use the formalism of Generalised Standard Materials (GSM) (Halphen, 1975) for which the constitutive equations can be derived from a thermodynamic potential and a dissipation potential.The chosen set of variables is $\{T, \varepsilon, \alpha\}, T$ being the temperature, $\varepsilon$ a strain tensor and $\alpha$ standing for all the other variables. The two principles of thermodynamics give the following local equations,

$$
\begin{aligned}
& \rho \dot{e}=\sigma: \dot{\varepsilon}+r_{e}-\operatorname{div} q \\
& \rho \dot{s}-\frac{r_{e}}{T}+\operatorname{div}\left(\frac{q}{T}\right) \geq 0
\end{aligned}
$$

where $e, \sigma, q, s, \rho$ and $r_{e}$ stand for the volume internal energy, the stress tensor, the heat influx vector, the specific entropy, the mass density and the external heat supply, respectively.

Classically, the total dissipation $d$ is introduced through the ClausiusDuhem inequality (3), and is generally split into an intrinsic dissipation $d_{1}$ and a thermal dissipation $d_{2}$. Each of these two dissipations is supposed to be non negative.

$$
\begin{aligned}
& \sigma: \dot{\varepsilon}-\rho e{ }_{\varepsilon} \dot{\varepsilon}-\rho e,_{\alpha} \dot{\alpha}-\frac{q}{T} \operatorname{gradT}=\frac{d}{T} \geq 0 \\
& d_{1}=\sigma: \dot{\varepsilon}-\rho e,_{\varepsilon} \dot{\varepsilon}-\rho e,_{\alpha} \dot{\alpha} \quad \text { and } \quad d_{2}=-\frac{q}{T} \operatorname{gradT}
\end{aligned}
$$

Using the formalism of GSM, a thermodynamic potential $\psi(T, \varepsilon, \alpha)$ (specific Helmholtz free energy) and a dissipation potential $\varphi(\operatorname{gradT}, \dot{\varepsilon}, \dot{\alpha})$ are introduced. Their derivatives with respect to the state variables and their time derivatives give the state equations (5a) and the evolution equations (5b).

$$
\text { a) }\left\{\begin{array} { l } 
{ \mathrm { s } = - \Psi , T } \\
{ \sigma _ { r } = \rho \Psi , \varepsilon } \\
{ \mathrm { A } = \rho \Psi , \alpha }
\end{array} \quad \text { b) } \left\{\begin{array}{l}
\mathrm{q}=\varphi, \text { grad } T \\
\sigma^{\text {ir }}=\varphi, \dot{\varepsilon} \\
\mathrm{A}=-\varphi, \dot{\alpha}
\end{array}\right.\right.
$$

In these last equations, $-A$ represents the thermodynamic force associated to the state variable $\alpha$, such as the product $-A \dot{\alpha}$ is the part of dissipated energy corresponding to the mechanism described by $\alpha$. 
The local heat conduction equation can be derived from the preceding expressions,

$$
\rho C \dot{T}+\operatorname{divq}=d_{1}+\rho T \psi,_{T \varepsilon}: \dot{\varepsilon}+\rho T \psi, T \alpha: \dot{\alpha}+r_{e}
$$

where $C$ denotes the specific heat capacity. Assuming a isotropic conduction law, (6) becomes

$$
\rho C \dot{\theta}-k \Delta \theta=w_{h}
$$

where $\theta$ is the temperature variation around the equilibrium temperature $T_{0},\left(\theta=T-T_{0}\right), k$ is the coefficient of thermal conduction and $w_{h}$ the volume heat source generated by all the terms in the right hand of equation (6).

This last term can be experimentally evaluated using temperature fields given by infrared thermography (see (Chrysochoos, 1995) fore more details). Another interesting experimental technique is the digital image correlation; it consists in correlating speckle images obtained by an optical camera to derive fields of displacement and strain. These two valuable techniques, providing thermal and mechanical fields, can be used to evaluate the various terms involved in $w_{h}$, and energy balances can be carried out.

This thermomechanical framework and these experimental devices have been used to investigate the behaviour of various materials: elastoplasticity in metallic alloys, thermovicoelasticity of polymers, phase change in shape memory alloys, localisation of thermomechanical fields (bands or necking), damage and unilateral effects in concrete for example. Two of these applications are detailed in this volume (Huon, 2001, and Muracciole, 2001).

We now focus on two particular studies where thermomechanical couplings have a great significance. The first one deals with Shape Memory Alloys and requires a multiscale approach, and the second one is concerned with analysing of thermal and dissipative effects associated with fatigue of metallic alloys.

\section{Multiscale approach of the thermomechanical behaviour of SMA}

SMA may undergo remarkable microstructural transformations: they may change the structure of their crystallographic lattice under mechanical and/or thermal loadings. This transformation, called martensitic transformation, is displacive in the sense that it corresponds to a collective displacement of atoms, and is considered as a first order phase transition described by a latent heat of phase change. Furthermore, the transition domain of SMA is generally close to the room temperature and thus is relatively easy to detect 

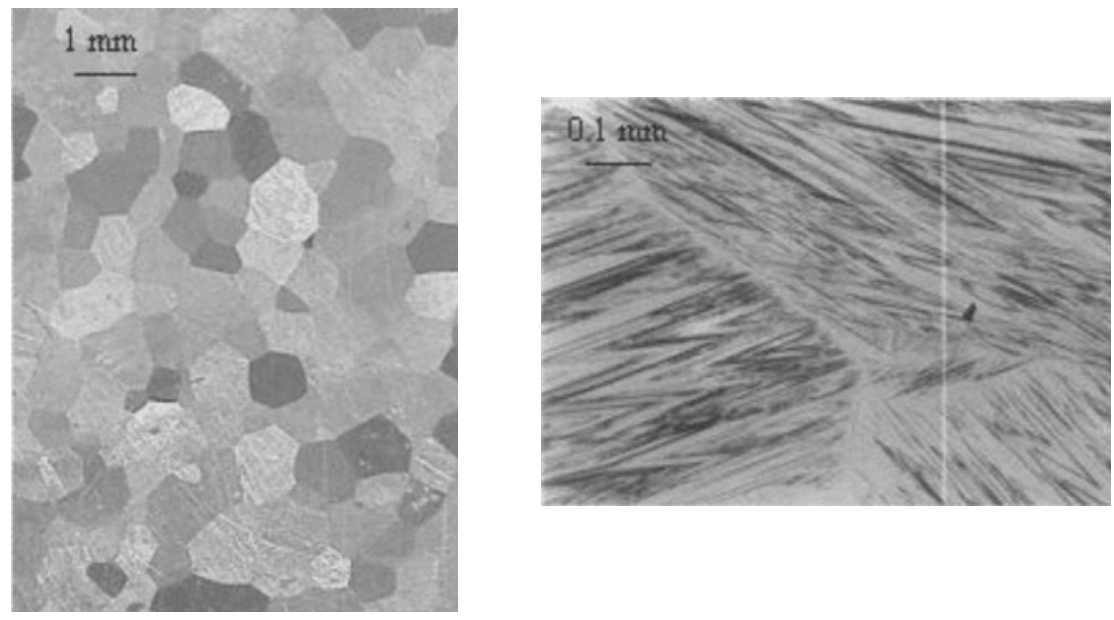

Fig. 1. Two characteristic views of a polycrystalline sample of CuZmAl defining the mesoscopic scale

and observe. Finally, and from a more practical point of view, the number of applications using SMA properties grows day after day.

One of the difficulties in the modelling of SMA behaviour consists in the choice of the description scale. In fact, three length scales can be introduced, starting from the crystallographic lattice level ( microscopic scale) up to the macroscopic scale of the sample, with an intermediate mesoscopic scale corresponding to the grain of the polycrystal or to the monocrystal (figure $1)$.

Some authors have systematically studied the potential microstructural arrangements (twinning) or rearrangements mechanisms due to straining (variants reorientation) when the material is in mechanical and thermodynamical equilibrium (Ball, 1987). Valuable results were obtained with good correlation with metallographic observations, but the connection with macroscopic behaviour seems difficult, especially when kinetic of phase change is to be modelled. Other authors (Auricchio, 1997, Frémond 1993, Lexcellent 1991, Patoor, 1987) were interested in the phase transition itself from a macroscopic point of view, that is pseudoelasticity, self-accommodation, one-way or two-way shape memory effects, but their works do not take into account neither microscopic considerations nor thermomechanical couplings due to phase transition. Following these works, we first present a phenomenological macroscopic model based on the analysis of thermomechanical couplings. 


\subsection{A first macroscopic analysis and modelling.}

This modelling (Peyroux, 1998) is based on experimental results, obtained from a thermomechanical device, which underline the main role played by the temperature variations induced by the deformation. The detailed analysis of the associated energy balances shows that the dissipated mechanical work (i.e. intrinsic dissipated energy) remains very small (less than $2 \%$ ) compared with latent heat of phase change, so that the temperature variations are essentially due to stress-induced phase transition.

On the basis of these results, a macroscopic modelling was proposed, that assumes an null intrinsic dissipation, takes account of thermomechanical couplings and, of course, considers anisothermal deformation processes.

For the sake of simplicity, only two self-accommodating variants of martensite are considered. Thus the set of state variables is $\left\{T, \varepsilon, x_{1}, x_{2}\right\}, x_{1}$ and $x_{2}$ being the volume fraction of the two variants. The strain tensor can be split into three parts, respectively due to elastic, thermal, and phase transition effects:

$$
\varepsilon=\varepsilon^{e l}+\alpha \theta I d+x_{v} \beta^{v}
$$

In equation $8, \alpha$ stands for the thermal expansion coefficient and the tensor $\beta_{v}$ characterises the phase change strain associated with the variant $v$. To ensure the self-accommodation property, we suppose $\beta_{1}=-\beta_{2}=\beta$, with $\operatorname{Tr}(\beta)=0$.

According to the experimental results, the dissipation potential $\varphi$ is identically equal to zero, and the thermodynamic potential is derived from the classical thermoelastic potential plus a specific part due to phase change $\psi_{c h}$.

$$
\rho \psi=\rho \psi_{e}+\rho \psi_{c h}+I_{v}\left(x_{1}, x_{2}\right) \text { and } \phi=0
$$

In equation 9 , the indicator function $I_{v}$ ensures physically admissible values to $x_{1}$ and $x_{2}$. Using the thermomechanical framework presented in section 2 , we can easily derived the constitutive equations:

$$
\begin{aligned}
& \sigma=a\left(\varepsilon-\alpha \theta-x_{v} \beta^{v}\right) \\
& \rho C \dot{\theta}-k \Delta \theta=-T_{0} a \alpha \dot{\varepsilon}+\rho L^{v} \dot{x}_{\nu}
\end{aligned}
$$

In equation 9, $a$ stands for the elasticity modulus tensor and in equation $10, L$ represents the latent heat of phase change. Furthermore, the nullity of the dissipation potential $\phi$ implies the nullity of the thermodynamic forces 
associated to $x_{1}$ and $x_{2}$, and thus gives supplementary inequalities connecting the stress tensor, the temperature and the volume fractions of martensite. This defines the transition domain (figure 2), giving the proportion of austenite $(A)$ and of the two variants of martensite $\left(M_{1}\right.$ and $\left.M_{2}\right)$.

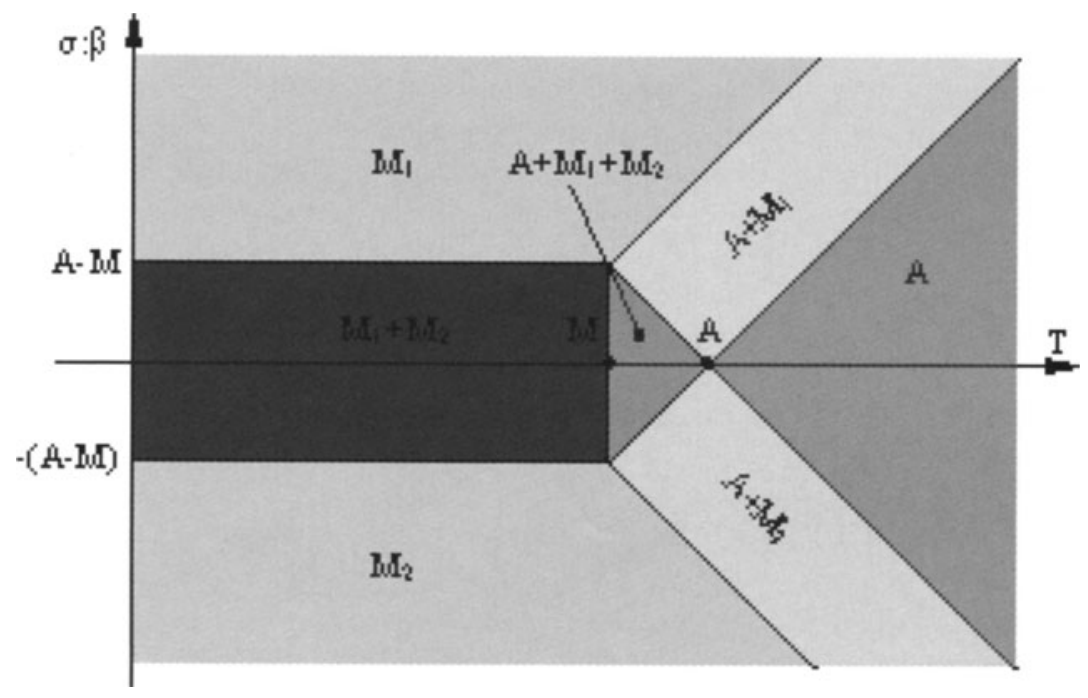

Fig. 2. Transition domain

This basic model gives a correct description of the main characteristic phenomena of SMA: pseudoelasticity, for high temperature behaviour, and reorientation effects for lower temperature. One of the most interesting results is in fact the prediction of temperature variations in the material. In agreement with the experimental results, these variations are found to be non negligible and of the same order of magnitude than the transition domain width. The taking into account of the anisothermal feature is then unavoidable and is the only way to predict accurately the transformation kinetic and the stress and temperature variations. As a direct consequence, the classical representation, in a stress-strain plane, of a pseudoelasticity test is not adequate, insofar as one can imagine several evolutions in the stress-straintemperature space giving the same projection in the stress-strain plane (see figure 3 ).

The main drawback of this purely macroscopic modelling is that it refers to volumic fractions of martensite, characterised by their orientation tensor $\beta$ which is tightly connected to the microscopic scale. To correct this lack of scale correlation, a scale transition can be used in order to derive the 
macroscopic behaviour from a mesoscopic model consistent both with the crystallographic data and the thermomechanical couplings. This mesoscopic modelling is described in the next part and the transition scale aspects will be presented in the following.

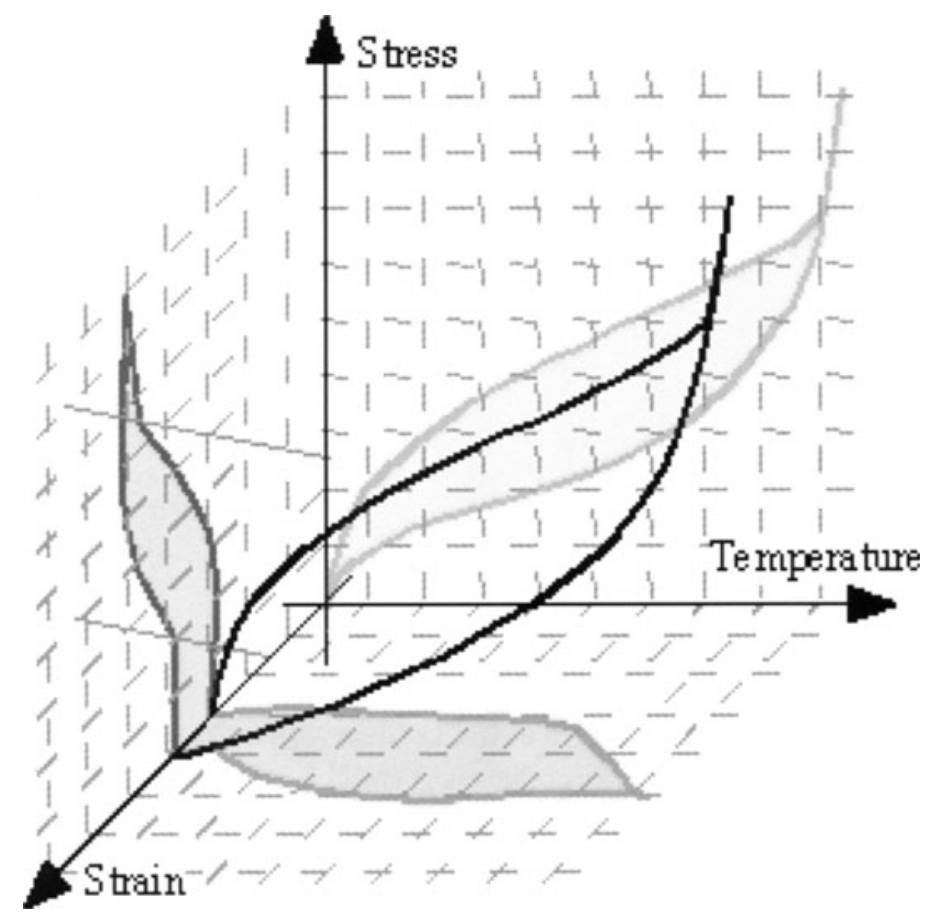

Fig. 3. Stress-strain-temperature evolution during a pseudoelasticity test

\subsection{A mesoscopic thermomechanical model}

In this part we aim to derive a set of constitutive equations appropriate for the behaviour of a grain of a polycrystal. To this end, we assume that each grain of the polycrystal behaves as a monocrystal and interacts with the adjoining grains. In the following, the mesoscopic model takes advantage of the crystallographic description of change phase at the microscopic level and of experimental results obtained on monocrystalline sample (Balandraud, 2000).

We consider the set of variables $\left\{\mathrm{T}, \varepsilon, x_{k}\right\}$, where $x_{k}$ stands for all the variants of variants of martensite required in the considered grain. The free energy $\Psi$ is chosen convex but not strictly convex with respect to $\varepsilon$ (figure 4) in order to provide a strain plateau under zero-stress (reorientation effect). 


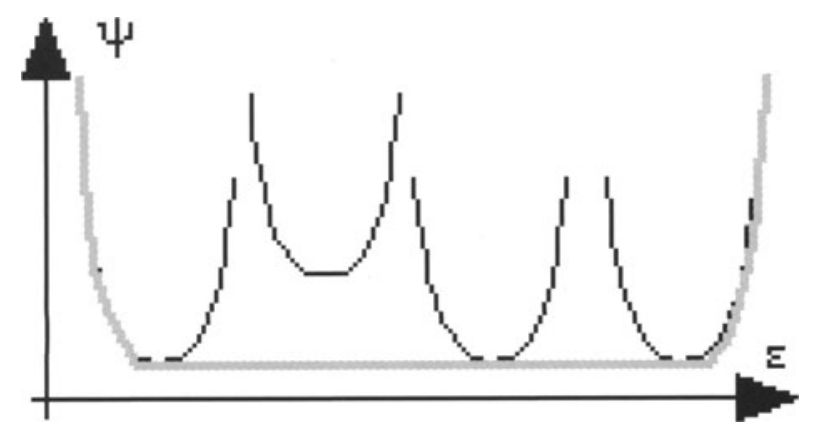

Fig. 4. Mesoscopic free energy

In fact it is more convenient here to deal with the marginal energy $\tilde{\psi}$, the definition of the transition domain resulting from this definition.

$$
\tilde{\psi}(T, \varepsilon)=\operatorname{Min}_{x_{k}} \psi\left(T, \varepsilon, x_{k}\right)
$$

Even if the thermal effects due to dissipation are difficult to measure, it is legitimate to suppose that a slight irreversibility accompanies the phase change. Consequently, we propose to chose the following dissipation potential:

$$
\phi=\phi\left(\dot{x}_{k}\right)=\frac{1}{2} K\left(\dot{x}_{k}^{2}\right)
$$

The existence of terms in $\dot{x}_{k}$ in the expression of the transition diagram expresses a dependence on the sense of transformation $(M \rightarrow A$ or $A \rightarrow M)$. Figure 5 shows the results of this modelling in terms of strain-strain relation and temperature evolution. The comparison with the non-dissipative case underlines that dissipation has a significant effect on the hysteresis area of the mechanical curve but a slight influence on the temperature evolutions.

Finally, this set of constitutive equations allows a correct prediction of pseudoelasticity, reorientation effect and recovery strain; it predicts phenomena such as asymmetry in tension-compression tests and time effects due to heat diffusion.

\subsection{Towards a macroscopic model}

We now focus on the macroscopic behaviour of a SMA polycrystalline aggregate. We again suppose that each grain behaves as a monocrystal and that the macroscopic behaviour can be derived from the thermomechanical 


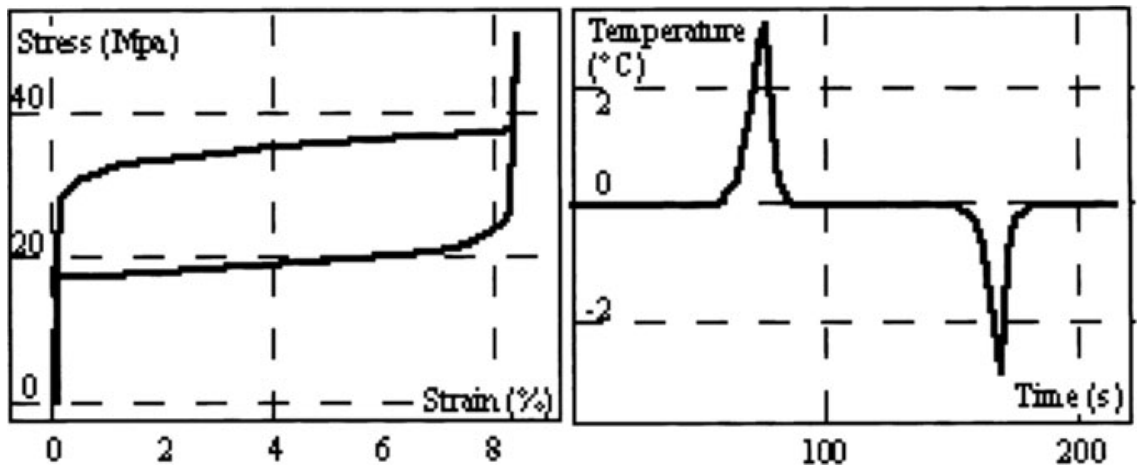

Fig. 5. Numerical stress-strain curve and temperature evolution in a pseudoelastic test

response of a representative volume element (RVE). The observation of a polycrystalline sample by means of electronic and optical microscopy, allows to gather data on the crystallographic texture, the shape and the statistical representation of the grains in the RVE (Figure 6). We also consider that the grains differ only by their crystallographic orientation.

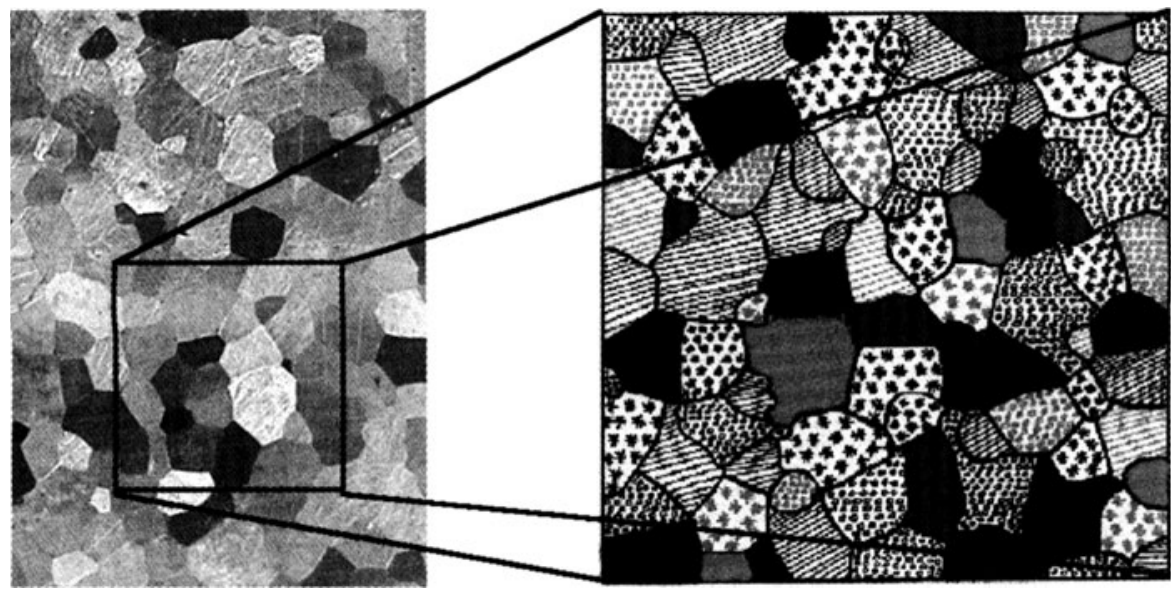

Fig. 6. RVE of a polycrystalline sample of CuZnAl SMA obtained by image analysis. Different patterns and colors stand for different crystallographic orientations

It is established, (Suquet, 1984) that for microscopic constitutive equations containing internal variables, "the homogenized law does not reduce to a single equation" on the macroscopic domain. "The knowledge of the macroscopic law requires as data the (mesoscopic) state variables". An alternative and pragmatic attitude is to link the macroscopic thermomechanical variables 
together, by prescribing thermomechanical loading and numerically solving the problems on the RVE. The RVE is considered as a virtual sample, and the finite element code as a virtual thermomechanical testing device.

The set of variables used at the mesoscopic level is $\left(T, \varepsilon, x_{k}\right)$, and additional variables such as the stress tensor $\sigma$ or the heat influx vector $q$ can be deduced from the initial set owing to the transition diagram or the heat equation.

On the macroscopic level, we consider the classic and natural macroscopic strain and stress tensors $E$ and $\Sigma$ defined as average values of $\varepsilon$ and $\sigma$ :

$$
E=<\varepsilon>\text { and } \quad \Sigma=<\sigma>
$$

In a first time we assume a quasi-homogeneous mesoscopic temperature field, the value of which is identified to the macroscopic temperature. Note that the quasi-homogeneity of the temperature field does not imply the homogeneity of its gradient.At this point $E^{p c}$, part of macroscopic strain due to phase change, is given by:

$$
E^{p c}=E-A^{\mathrm{hom}^{-1}} \Sigma-\alpha^{\mathrm{hom}} \theta
$$

where $A^{\text {hom }}$ is the macroscopic tensor of elasticity, and $\alpha^{\text {hom }}$ the macroscopic thermal expansion coefficient.

It remains to define $X$ : macroscopic equivalence to the volume proportion of phase change. Deriving $\bar{L}$, macroscopic latent heat, from $\bar{\rho} \bar{L}=\langle\rho L\rangle$, we can propose as a definition of $X$ :

$$
\bar{\rho} \bar{L} X=\langle\rho L x\rangle
$$

This macroscopic proportion has to be regarded as an energy indicator of the advancement of phase change. The value of $X$ is 0 if the polycrystal is completely austenitic, and reaches 1 if completely martensitic. However a value between 0 and 1 can be reached under several microscopic configurations.

Then, it is of some interest to express $E^{p c}$ as:

$$
E^{p c}=X \beta^{\text {hom }}
$$

where $\beta^{\text {hom }}$ represents the maximum potential strains associated to phase change.

A convenient set of macroscopic variables being established, we finally present an example of the results of finite element simulations on the RVE (Balandraud, 2000).

We numerically performed a pseudoelasticity test consisting of a loadunload path at a room temperature upper than $T_{A}$ and the different results 


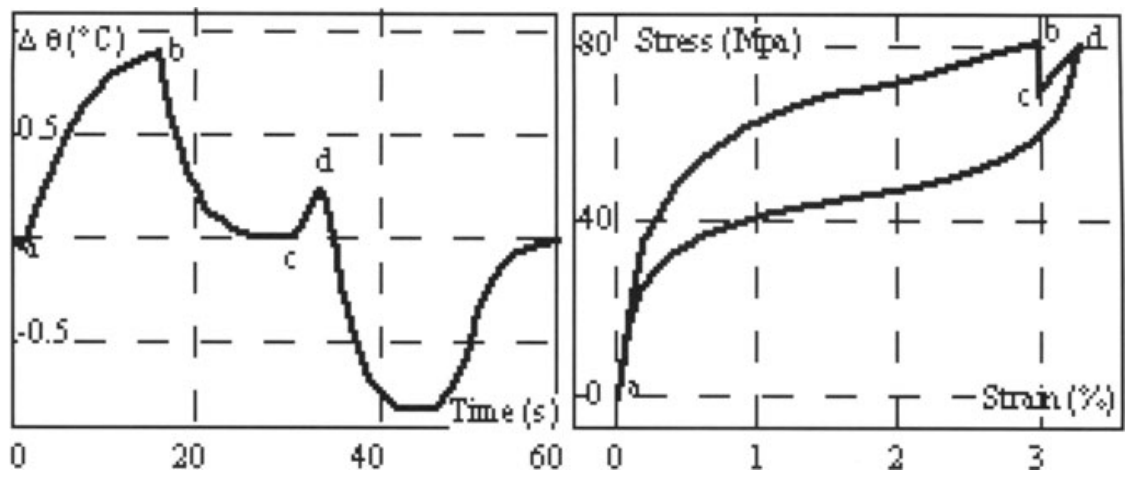

Fig. 7. Results of the pseudoelastic numerical test

are plotted in figure 7. The imposed strain is increased from point $a$ to point $d$, and maintained at a certain level between points $b$ and $c$. In the particular stage $b c$, we can observe the role played by thermomechanical couplings. The material keeps on transforming ( $X$ increases), the stress relaxes while the temperature returns to the imposed value. Concerning the entire test, the evolution of the different variables is consistent with experimental results obtained on polycrystalline SMA. This macroscopic behavior is of course different from the monocrystalline one and corresponds to the particular ordering of grains chosen in the RVE.

The possibilities of this approach have been widely developed in (Balandraud, 2000). For instance, low-temperature tests allowed the determination of the value of the macroscopic recoverable strain due to variant reorientation at zero stress. This numerical tool was of great use when investigating multiaxial behaviour of polycrystalline SMA and when studying the influence of various dissipation potentials.

\section{Thermal and dissipative effects associated with fatigue of metallic alloys.}

Statistical considerations on time-consuming and expensive tests are needed to evaluate the fatigue resistance of materials and structures. Increasing pressure for shorter design assessment time in industry leads researchers to develop new short-time methods of fatigue limit determination.

In this aim, variations of specimen temperature during fatigue tests have been studied (Bousseau, 1998, Luong, 1998, La Rosa, 2000, Liaw, 2000, Galtier, 2001). These observations incited researchers to propose a new methodology for the determination of fatigue limit. This methodology is based on the analysis of thermal effects associated with fatigue testing. However, some of its application are still problematic. Indeed, the observed heating does not 
reveal intrinsic material behaviour as it depends on various parameters such as heat diffusion.

Therefore, we present in the following a calorific approach of fatigue phenomenon rather based on heat sources analysis than on thermal effects study. The aim is to evaluate dissipation associated with the observed heating and to examine the possible couplings between thermoelasticity and fatigue.

\subsection{Thermal effects associated with fatigue testing}

Preliminary observations Since years 30, fatigue specimen temperature variations have received great interest. These temperature variations have been measured either by thermocouples or, more recently, using infrared thermography. All the authors (Bousseau, 1998, La Rosa, 2000, Liaw, 2000) agreed on the following observations :

-the greater the applied stress and the frequency, the higher the temperature increase during fatigue test.

-for given stress and frequency, the average specimen temperature increases during the first part of the test (first thousands cycles), then remains constant (steady-state conditions) until an abrupt increase immediately prior to failure.

This suggests that, concerning temperature, the few first thousands cycles are relevant and leads to consider this steady-state temperature as a representative parameter to characterize fatigue.

\section{Short-time measurements of fatigue limit using thermal analysis}

Some authors derived a new method for the determination of the fatigue limit from these preliminary observations. This methodology is based on the evolution of the steady-state temperature as a function of the applied stress amplitude (see (La Rosa, 2000, Luong 1998) for detailed procedure). A specimen is successively loaded at different stress levels for about $10^{4}$ cycles. For each loading level, the steady-state temperature $\Delta \bar{T}$ (supposed to be reached after $10^{4}$ cycles) is measured. The development of $\Delta \bar{T}$ the steady-state temperature, plotted as a function of the stress level, exhibits a well-marked break. The stress amplitude corresponding to this break appears to be close to the fatigue limit as evaluated with usual methods.

This new method offers considerable interest compared to usual techniques: it allows to obtain reliable results using a very limited number of specimens in a very short time. In addition, it is easily applicable to structures. Consequently, this methodology, which validity has been confirmed by different applications, has been adopted in industrial development (Bérard, 1998). However, some applications, concerning aluminium alloy for instance, are still critical. Indeed, in that case, the steady-state temperature does not show a clear evolution with respect to stress levels. Hence, it is impossible to establish an obvious relation between temperature variations and fatigue limit. 
Anyway, even if in most cases, temperature seems to be a relevant parameter to characterize irreversibilities that occur during fatigue testing, it does not express the intrinsic behaviour of the material. Actually, temperature depends on the specimen geometry, on the external heat exchange conditions and on the homogeneity of the studied phenomenon. In order to take into account these various structural effects, we propose to analyse the fatigue mechanisms considering heat sources instead of temperature.

\subsection{Dissipation and couplings}

The set of state variables to be used in the above-mentioned thermomechanical framework is $(T, \varepsilon, f)$, where $f$ is a scalar parameter standing for the state of fatigue in the material.

The local heat conduction equation is then :

$$
\rho C \dot{\theta}-k \Delta \theta=d_{1}+\rho T \psi,_{T \varepsilon}: \dot{\varepsilon}+\rho T \psi,_{T f}: \dot{f}
$$

Supposing the mechanical irreversibilities only stem from fatigue effects (i.e. no irreversible stress), the intrinsic dissipation can be written:

$$
d_{1}=-\rho \psi,_{f \dot{f}}
$$

Assuming that the slight temperature variations $\left(\theta<<T_{0}\right)$ does not affect the state of fatigue but, on the contrary, that the state of fatigue can modify the thermoelastic response of the material, we can write:

$$
\begin{aligned}
& \psi,_{T f}=0 \\
& \psi,_{T \varepsilon}=b(f)
\end{aligned}
$$

In the case of a one-dimension thermoelastic behaviour including fatigue state variable, the strain becomes:

$$
\varepsilon=\frac{\sigma}{E(f)}+\alpha(f) \theta
$$

where $E$ is the Young modulus, and $\alpha$ the thermal expansion coefficient. Equations (20) and (22) lead to:

$$
(E \alpha)_{f}=0
$$


that is to say $b(f)$ is constant. So, when $E$ decreases, $\alpha$ increases, and the strain amplitude gets greater as the fatigue process progresses. Thermoelastic sources amplitude will consequently increase:

$$
\Delta S_{\text {the }}=T_{0} E \alpha \Delta \dot{\varepsilon}
$$

Then, it is possible to estimate the thermoelastic sources amplitude for several loading cycles, supposing the evolution of $f$ is slow, that is the variations of $f$ can be neglected. Steady-state temperature reached after a load step of some thousands cycles will be associated with average dissipation.

\subsection{Experimental}

Fatigue tests were performed on a servohydraulic machine with a $25 \mathrm{kN}$ load cell. These tests were conducted at $10 \mathrm{~Hz}$ with a load ratio $\mathrm{R}=0.1$. Successive load steps of $10^{4}$ cycles were carried out on a same specimen. Surface temperature images of the tested sample were provided using an infrared CCD camera (short waves, NETD $\sim 25 \mathrm{mK}$ ). High-speed data acquisition capabilities are available at $50 \mathrm{~Hz}$ with a full-frame $\left(256^{*} 256\right.$ pixels $)$ and at $180 \mathrm{~Hz}$ with a narrow window $\left(128^{*} 128\right.$ pixels). An infrared image processing has been developed in order to deduce the distribution of heat sources from the surface temperature measurements by the mean of equation (18). This data processing is based on local least-square fitting (Wattrisse, 2000). Hypothesis needed for its application as well as validation checking are also presented in (Chrysochoos, 2000).

Specimens were flat $(1 \mathrm{~mm}$ thick): this allows to consider the surface temperature as the average thick-wise temperature. All specimens were coated with a black spay paint to maximise their emissivity. In addition, the paint coating reduces surface reflections and ensures uniformity of the specimen response.

A stainless steel (304L) and an aluminium alloy (AU4G) were studied. For these two materials, thermophysical characteristics needed to determine heat sources are given in table 1 .

\begin{tabular}{|c|c|c|c|c|c|}
\hline & $\rho\left(\mathrm{kg} \cdot \mathrm{m}^{-3}\right)$ & $\mathrm{C}\left(\mathrm{J} \cdot \mathrm{kg}^{-1} \cdot \mathrm{K}^{-1}\right)$ & $\tau_{t h}(s)$ & $\tau_{e q}(s)$ & $\mathrm{k}\left(\mathrm{W} \cdot \mathrm{m}^{-1} \cdot \mathrm{K}^{-1}\right)$ \\
\hline $304 \mathrm{~L}$ & 7900 & 480 & 327 & 45 & 60 \\
\hline $\mathrm{AO} 4 \mathrm{G}$ & 2800 & 930 & 225 & 18 & 175 \\
\hline
\end{tabular}

Table 1 : Thermophysical characteristics of the materials

$\tau_{t h}$ is a time constant related to the heat losses and characterises the lateral heat exchanges between the sample and the surrounding air. If the heat sources are assumed to be uniform, the laplacian operator can be reduced to a linear term. An equivalent time constant $\tau_{e q}$ is then defined :

$$
\frac{1}{\tau_{e q}}=\frac{1}{\tau_{t h}}+\frac{k}{\rho C}\left(\omega_{0}^{2}+\Omega_{0}^{2}\right)
$$


where $\omega_{0}$ et $\Omega_{0}$ are the first eigen-pulsations of the laplacian operator (Chrysochoos, 2000) In that case, steady-state temperatures are related to the average intrinsic dissipation:

$$
\Delta \bar{T} \approx \frac{\tau_{e q}}{\rho C} \bar{d}_{1}(\dot{f})
$$

Besides, temperature variations amplitude for a load cycle is:

$$
\Delta T \approx-\frac{\alpha(f) T_{0}}{\rho C} \frac{4 \pi f_{r}}{\sqrt{\tau_{e q}^{-2}+4 \pi^{2} f_{r}^{2}}} \sigma_{0}
$$

with $f_{r}$ standing for the loading frequency. These temperature variations over one cycle are due to thermoelastic coupling.

\subsection{First experimental results}

During a load step (figure 8), the specimen shows on the one hand an average temperature increase related to energy dissipation, and, on the other hand, sinusoidal fluctuations around this average value. If all other couplings are neglected as supposed in $\S 4.2$, these fluctuations are induced by thermoelastic effects.

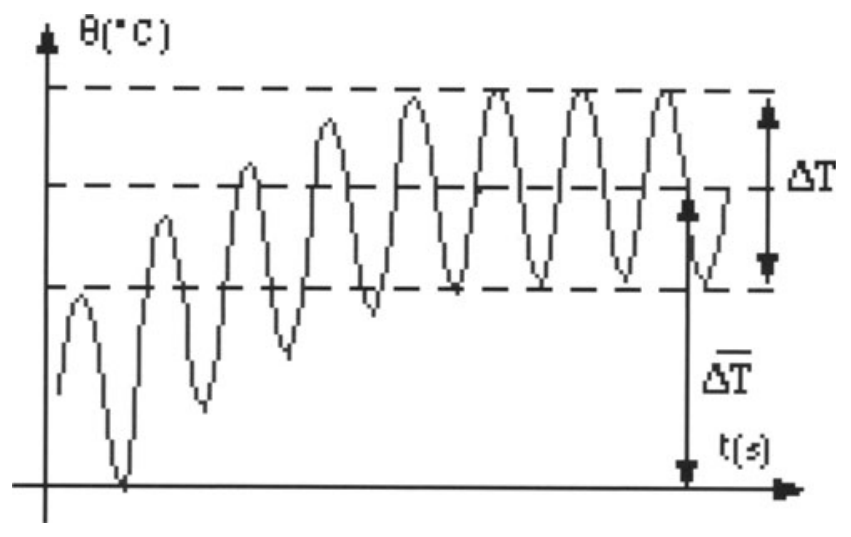

Fig. 8. Experimental evolution of temperature during a fatigue cyclic loading

Figure 9 presents results obtained for the whole load steps in the case of two stainless steel specimens. Steady-state temperature evolution is fully 


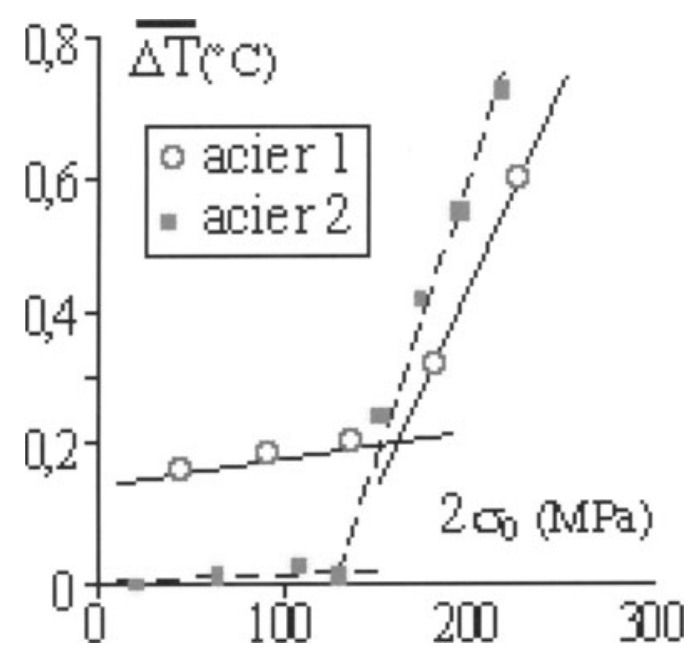

Fig. 9. Evolution of $\Delta \bar{T}$ for the A304L steel

consistent with literature results: a well-marked break in the curves is observed.

From a calorific point of view, (Figure 10), thermoelastic sources amplitude evolution is linear for the first load steps, as expected in classical thermoelasticity (constant Young modulus and constant thermal expansion coefficient). After these first load steps, a clear change is noted and might be attributed to coupling mechanisms between fatigue and thermoelasticity (cf. equation 27).

This change in the thermoelastic sources amplitude evolution occurs at the same stress level as for temperature curves break. At last, it is worth noting that average dissipations $\bar{d}_{1}$ are about $10^{3}$ times lower than thermoelastic sources for a loading frequency $f_{r}=10 \mathrm{~Hz}$. These average dissipations have been estimated using equation 26 .

The results obtained on two aluminium alloy specimens are shown in figure 11. The lack of clear trend when plotting average dissipation (i.e. steadystate temperature, see equation 26) versus stress level is confirmed for this type of material. However, thermoelastic sources development is much more regular and exhibits a marked break for one of the specimens, as it was observed for stainless steel.

It must be noted that the second specimen has been tested at the last stress level for more than $10^{7}$ cycles without failing. This, combined with the strictly linear development of sources amplitude, suggests the fatigue limit has not been reached in that case. 

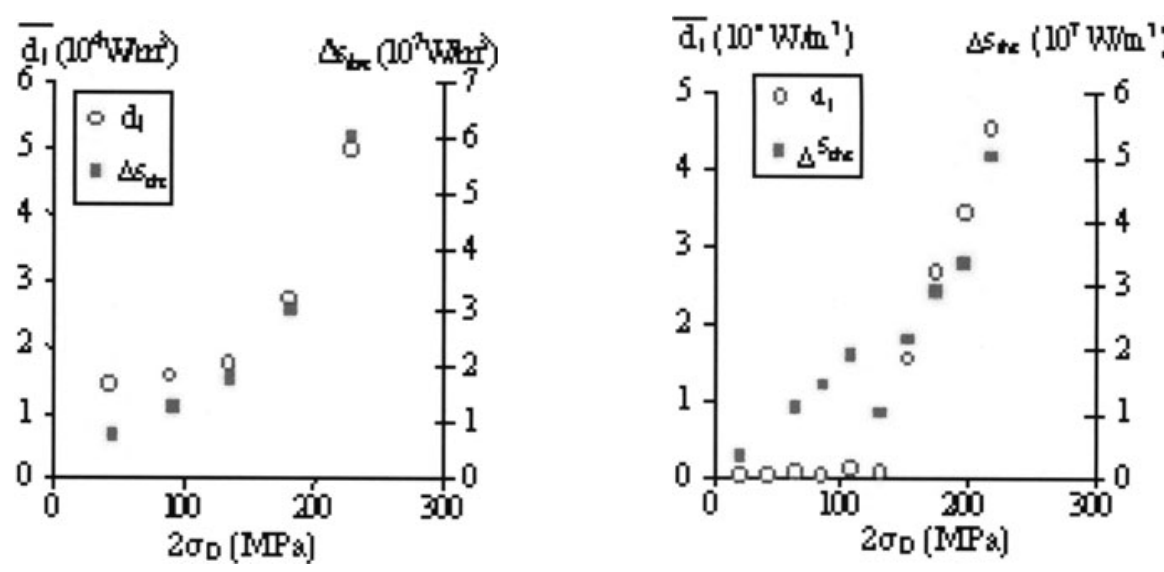

Figure 10: Average dissipation and thermoelastic sources amplitude for $\mathrm{A} 304 \mathrm{~L}$ steel
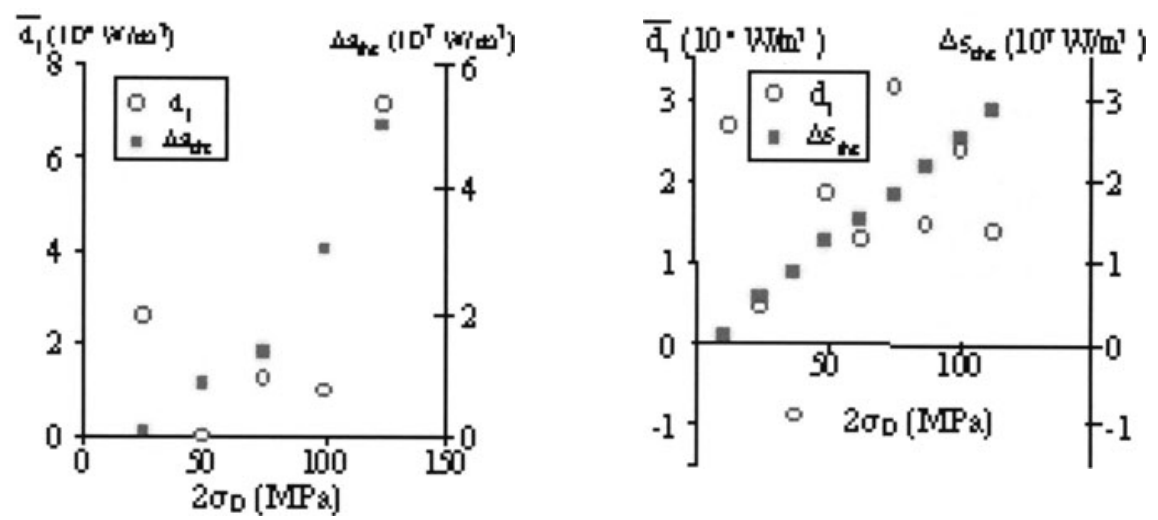

Figure 11: Average dissipation and thermoelastic sources amplitude for aluminium alloy

\subsection{Prospects}

The scattering of these first results provided by a few tested specimens must be considered from the statistical point of view associated with fatigue testing. It would be premature to point out any conclusion. Nevertheless, the analysis of fatigue effects via thermoelastic sources amplitudes appears to be attractive. For instance, in the case of aluminium alloys, the data are more consistent when reported in terms of sources amplitudes than in terms of temperature.

It would be of high interest to develop the same kind of approach considering 
not only average dissipation for a load step but also instantaneous dissipation. This could be performed by reducing significantly thermal drift during the whole test or by increasing load frequency. Concerning modelling, thermoelastic sources and dissipation are related respectively to the variable $f$ and its time derivative. These independent and complementary calorific data could be used to identify this fatigue variable and its evolution law.

\section{References}

1. Auricchio, F., Lubliner, J., 1997; A uniaxial model for shape memory alloys, Int. J. of Solids and Structures, Vol. 33, pp. 3601-3618.

2. Balandraud, X., 2000; Changement de phase et changements d'é chelle dans les alliages à mémoire de forme, Thesis of University Montpellier II.

3. Ball, J.M., James, R., 1987; Fine phase mixture as minimizers of energy, Arch. Ration. Analysis, 100, pp. 15-52

4. Bérard, J.Y., Rathery, S., Béranger, A.S., 1998; Dé termination de la limite d'endurance des matériaux par thermographie infrarouge. Application sur un bras de suspension. , Matériaux et Techniques, 1-2, 55-57.

5. Bousseau, M., 1998; Application de la thermographie infrarouge au suivi de l'endommagement de fatigue, PhotoMécanique 98, 199-206.

6. Chrysochoos, A., 1995; Analyse du comportement thermomé canique des matériaux par thermographie infrarouge, Photomécanique 95, Ed. Eyrolles, pp.203-211.

7. Chrysochoos, A., Louche, H., 2000; An infrared image processing to analyse calorific effects accompanying strain localisation, Int. J. of Eng. Sci., 38, 17591788 .

8. Frémond, M., 1993; Matériaux à mémoire de forme, C. R. Acad. Sc. Paris, série II, Vol. 316, pp.1031-1036.

9. Galtier A., 2001; Influence de la microstructure des aciers sur leurs propriétés mécaniques, Colloque MECAMAT - Aussois 2001, 55-60.

10. Germain, P., 1973; Cours de Mécanique des Milieux Continus, Masson et Cie Ed., p. 417

11. Germain,P., Nguyen Q.S., Suquet, P., 1983; Continuum Thermodynamics, J. of Appl. Mech., Transactions of the ASME, 50, pp. 1010-1020.

12. Halphen, B., Nguyen Q.S., 1975; Sur les Matériaux Standards Généralisés, Journal de Mécanique, Vol. 14, 1.

13. Huon, V., Cousin, B., Maisonneuve, O., 2001; Study of thermal and kinematic phenomena associated with quasi-static deformation and damage process of some concretes, this volume.

14. La Rosa, G., Risitano, A., 2000; Thermographic methodology for rapid determination of the fatigue limit of materials and mechanical components, International Journal of Fatigue, 22, 1, 65-73.

15. Lexcellent, C., Licht, C., 1991; Some remarks on the modelling of the thermomechanical behaviour of shape memory alloys, J. de Physique, colloque C4, vol. 1, pp. 35-39.

16. Liaw, P. K., Wang, H., Jiang, L., Yang, B., Huang, J.Y., Kuo, R.C., Huang, J.C., 2000; Thermographic detection of fatigue damage of pressure vessel steels at $1000 \mathrm{~Hz}$ and $20 \mathrm{~Hz}$, Scripta Materialia, 42, 4, 389-395. 
17. Luong, M. P., 1998; Fatigue limit evaluation of metals using an infrared thermographic technique, Mechanics of Materials, 28, 155-163

18. Muracciole, J.M., Wattrisse, B., Chrysochoos, A., 2001; Local experimental analyses of the thermomechanical heterogeneous behaviour of thermoplastics, this volume.

19. Patoor, E., Eberhardt, A., Berveiller, M., 1987; Potentiel pseudoélastique et plasticité de transformation martensitique dans les mono et polycristaux métalliques. Acta Metall., Vol. 35, pp. 2779-2789.

20. Peyroux, R., Chrysochoos, A., Licht, C., Löbel, M., 1998; Thermomechanical couplings and pseudoelasticity of shape memory alloys, Int. J. of Engng. Sci., Vol. 36, $\mathrm{n}^{\circ} 4$, pp. 489-509. 